\title{
Licochalcone A induces apoptosis in malignant pleural mesothelioma through downregulation of Sp1 and subsequent activation of mitochondria-related apoptotic pathway
}

\author{
KA HWI KIM ${ }^{1}$, GOO YOON ${ }^{1}$, JUNG JAE CHO ${ }^{1}$, JIN HYOUNG CHO ${ }^{2}$, \\ YOUNG SIK CHO ${ }^{3}$, JUNG-IL CHAE ${ }^{2}$ and JUNG-HYUN SHIM ${ }^{1}$ \\ ${ }^{1}$ Natural Medicine Research Institute, Department of Pharmacy, College of Pharmacy, Mokpo National University, \\ Jeonnam 534-729; ${ }^{2}$ Department of Oral Pharmacology, School of Dentistry and Institute of Oral Bioscience, \\ BK21 plus, Chonbuk National University, Jeonju 651-756; ${ }^{3}$ College of Pharmacy, Keimyung University, \\ 1000 Sindang-dong, Dalseo-gu, Daegu 704-701, Republic of Korea
}

Received October 13, 2014; Accepted December 2, 2014

DOI: 10.3892/ijo.2015.2839

\begin{abstract}
Licochalcone A (LCA) is a natural product derived from the roots of Glycyrrhiza inflata exhibiting a wide range of bioactivities such as antitumor, anti-oxidant and anti-bacterial effects. Malignant pleural mesothelioma (MPM) is an extremely aggressive type of cancer with a poor prognosis because of its rapid progression. However, LCA has not been investigated concerning its effects on MPM. Preliminarily, we observed that LCA negatively modulated not only cell growth, but also specificity protein 1 (Sp1) expression in MSTO-211H and $\mathrm{H} 28$ cell lines. It was found that $\mathrm{IC}_{50}$ values of LCA for growth inhibition of MSTO-211H and $\mathrm{H} 28$ cells were approximately 26 and $30 \mu \mathrm{M}$, respectively. Consistent with downregulation of Sp1, expression of Sp1 regulatory proteins such as Cyclin D1, Mcl-1 and
\end{abstract}

Correspondence to: Professor Jung-Il Chae, Department of Oral Pharmacology, School of Dentistry, BK21 plus, Chonbuk National University, Jeonju 561-756, Republic of Korea

E-mail: jichae@jbnu.ac.kr

Professor Jung-Hyun Shim, Department of Pharmacy, College of Pharmacy, Mokpo National University, 1666 Youngsanro, Muan-gun, Jeonnam 534-729, Republic of Korea

E-mail: s1004jh@gmail.com

Abbreviations: MPM, malignant pleural mesothelioma; LCA, licochalcone A; Sp1, specificity protein 1; RPMI-1640, Roswell Park Memorial Institute; FBS, fetal bovine serum; PBS, phosphate-buffered saline; Mcl-1, myeloid cell leukemia-1; PARP, poly(ADP-ribose) polymerase; MTS, (3-(4,5-dimethylthiazol-2-yl)-5-(3-carboxymethoxyphenyl)-2-(4-sulfophenyl)-2H-tetrazolium); DAPI, 4'-6-diamidino-2phenylindole; PI, propidium iodide; LCE, licochalcone E; TNF, tumor necrosis factor; TRAIL, TNF-related apoptosis-inducing ligand

Key words: licochalcone A, apoptosis, specificity protein 1, malignant pleural mesothelioma, mitochondria
Survivin was substantially diminished. Mechanistically, LCA triggered the mitochondrial apoptotic pathway by affecting the ratio of mitochondrial proapoptotic Bax to anti-apoptotic $\mathrm{Bcl}_{\mathrm{xL}}$. Bid induced loss of mitochondrial membrane potential, eventually leading to multi-caspase activation and increased sub- $\mathrm{G}_{1}$ population. Moreover, nuclear staining with DAPI highlighted nuclear condensation and fragmentation of apoptotic features. Flow cytometry analyses after staining cells with Annexin V and propiodium iodide corroborated LCA-mediated apoptotic cell death of MPM cells. In conclusion, these results present that LCA may be a potential bioactive material to control human MPM cells by apoptosis via the downregulation of Sp1.

\section{Introduction}

Cancer is a major public health problem in the United States as well as many other countries (1). It is the second leading cause of death after heart disease, one quarter of Americans dying from cancers. Billions of dollars are spent every year for treatment and prevention of cancer (2). Among cancers, malignant pleural mesothelioma (MPM), which occurs in the peritoneum and pleura, is rare but progressive and rapidly fatal (3). The onset has been related to exposure to occupational and environmental asbestos (4). The disease often develops in male and the incidence increases with age (3). Diagnosis or management for MPM has not been precisely defined. Diagnosis is determined from the immunocytochemical analysis and histological examination of biopsy or diagnostic laparoscopic specimens (3). Median survival time is only 5-12 months and mean symptoms-to-survival time is 345 days due to lack of effective treatment $(5,6)$. Other treatments are possible, but local excision and radical resection are preferred $(7,8)$. Chemotherapy has an important role in the palliative treatment. Recently, application of natural products has gained attention as cancer chemopreventive therapy (9). Licorice root is a common source of licorice used in traditional and herbal medicines. The pharmacological activity of licorice has been used for the treatment of human diseases such as cancer, gastric ulcers, bacterial infections and immunodeficiency (10). LCA 
is a flavonoid extracted from licorice and exerts anti-tumor properties, anti-parasites and anti-microbial activity (11-13). LCA inhibits TNF- $\alpha$-induced nuclear factor- $\kappa \mathrm{B}$ activation, leading to downregulation of inflammatory cytokines with concomitant reduction of carrageenan-induced inflammatory responses $(14,15)$. However, it remains unclear whether LCA induces apoptosis by suppressing the expression of specificity protein $1(\mathrm{Sp} 1)$ in human MPM cells.

$\mathrm{Sp} 1$ is one of the first eukaryotic transcription factors to be identified and cloned and it is ubiquitously expressed in mammalian cells $(16,17)$. It is also a sequence-specific DNA-binding protein and plays an important role in various physiological processes, including angiogenesis, cell cycle regulation, hormonal activation and apoptosis (18). In many studies, the expression levels of Sp1 are higher in cancer cells such as human pancreatic cancer, breast cancer, colorectal cancer, gastric carcinoma, hepatocellular carcinoma and thyroid carcinoma than in normal cells (19). Further, there is also evidence that $\mathrm{Sp} 1$ protein functions as an important factor in the development of tumors, growth and metastasis (17). However, involvement of Sp1 protein in the pathogenesis of MPM is unknown. In order to verify its therapeutic potential, we investigated whether LCA could induce apoptosis by suppressing Sp1 protein level in MSTO-211H and H28 cells.

\section{Materials and methods}

Plant material. The roots of Glycyrrhiza inflata were purchased from Chonnam Herb Association. A voucher specimen (MNUYG-003) was deposited in the College of Pharmacy, Mokpo National University, Muan, Korea.

Extraction and isolation. The air-dried, powdered G. inflata roots $(600 \mathrm{~g})$ were extracted twice with $\mathrm{MeOH}(41)$ by sonication for $3 \mathrm{~h}$. After filtration, the $\mathrm{MeOH}$ extract was evaporated and suspended in distilled water and then defatted with $n$-hexane (1 1). The aqueous layer was partitioned with methylene chloride $(3 \times 11)$. The evaporation residue $(5 \mathrm{~g})$ was subjected to flash silica gel chromatography, using $n$-hexane:EtOAc:MeOH solvent system (2:1:0.1-1:1:0.1-100\% MeOH), to afford 10 fractions. Fractions 2, 3 and 4 were subjected to further flash silica gel chromatography, with a chloroform:MeOH (100:1) eluent system, to afford LCA (50 mg). LCA was further purified by column chromatography using RP18 to an analytically acceptable purity.

Cell culture. The human MPM cells MSTO-211H and H28 were purchased from the American Tissue Culture Collection (Manassas, VA, USA) The MSTO-211H and H28 cells were grown in RPMI-1640 medium, supplemented with $10 \%$ fetal bovine serum, $2 \mathrm{mM}$ L-glutamine, $100 \mathrm{U} / \mathrm{ml}$ each of penicillin and streptomycin (Thermo Scientific, Logan, UT, USA) at $37^{\circ} \mathrm{C}$ in a humidified atmosphere of $5 \% \mathrm{CO}_{2}$ and $95 \%$ air.

MTS assay. Effects of LCA on the cell viability were determined using the MTS assay according to the manufacturer's instructions. The cells were plated on a 96 -well plate at a density of $8 \times 10^{4}$ cells/well for MSTO-211H and $3 \times 10^{4}$ cells/well for $\mathrm{H} 28$ and then treated with various concentrations of LCA for
24 and $48 \mathrm{~h}$. After addition of MTS solution to culture media, the absorbance was measured at $490 \mathrm{~nm}$ using an Enspire Multimode Plate reader (Perkin-Elmer, Akron, OH, USA). The data were expressed as the percentage of cell viability compared with the control.

DAPI staining. The number of cells undergoing apoptosis, which was induced by LCA treatment, was quantified using DAPI staining. The cells featuring nuclear condensation and fragmentation were determined using nucleic acid stained with 4'-6-diamidino-2-phenylindole (DAPI) (Sigma-Aldrich). Forty-eight hours after treatment with different doses of LCA $(10,20$ and $40 \mu \mathrm{M})$, the MSTO-211H and H28 cells were harvested and fixed in $100 \% \mathrm{MeOH}$ at RT for $20 \mathrm{~min}$. The cells were seeded on slides, stained with DAPI $(2 \mu \mathrm{g} / \mathrm{ml})$ and then analyzed under a FluoView confocal laser microscope (Fluoview FV10i, Olympus Corp., Tokyo, Japan).

Cell cycle analysis. Following treatment of the cells with LCA for $48 \mathrm{~h}$, the detached cells and adherent cells were collected separately. The cells were washed with cold PBS and then centrifuged before being fixed in $70 \% \mathrm{EtOH}$ for $3 \mathrm{~h}$ at $-20^{\circ} \mathrm{C}$. Before flow cytometry analysis, the cells were centrifuged and incubated for $30 \mathrm{~min}$ at $37^{\circ} \mathrm{C}$ in PBS to allow for the release of low-molecular weight DNA. Following centrifugation, the cell pellets were suspended and treated with Muse ${ }^{\mathrm{TM}}$ cell cycle reagent using a Muse ${ }^{\mathrm{TM}}$ Cell Analyzer (Merck Millipore, Billerica, MA, USA).

Reverse-transcription polymerase chain reaction (RT-PCR). Total RNAs were isolated using TRIzol ${ }^{\circledR}$ Reagent (Life Technologies, Carlsbad, CA, USA) and $2 \mu \mathrm{g}$ of RNA was used to synthesize cDNA using the HelixCript ${ }^{\mathrm{TM}}$ 1st-strand cDNA synthesis kit (NanoHelix). cDNA was obtained by PCR amplification using $\beta$-actin-specific and Sp1-specific primers using the following PCR conditions: 35 cycles of $1 \mathrm{~min}$ at $95^{\circ} \mathrm{C}, 1 \mathrm{~min}$ at $56^{\circ} \mathrm{C}$ and $1 \mathrm{~min}$ at $72^{\circ} \mathrm{C}$. The $\beta$-actin primers used were: forward, 5'-GTG-GGG-CGC-CCC-AGG-CAC-CA-3'; and reverse, 5'-CTC-CTT-AAT-GTC-ACG-CAC-GAT-TTC-3'. The Sp1 primers used were: forward, ATG CCT AAT ATT CAG TAT CAA GTA; and reverse, CCC TGA GGT GAC AGG CTG TGA. PCR products were analyzed by $2 \%$ agarose gel electrophoresis.

Mitochondrial membrane potential assessment. To investigate the mitochondrial membrane permeability, MSTO-211H and $\mathrm{H} 28$ cells were cultured with LCA at given doses for 48 h. After washing with PBS, cells were dissociated by trypsin. For detection of the depolarized mitochondria of cells undergoing apoptosis, MitoPotential working solution to the MPM cells was added for 20 min incubation in the dark. Muse MitoPotential 7-AAD reagent was added to each well and then kept in the dark for $5 \mathrm{~min}$. The experiment was analyzed by Muse ${ }^{\mathrm{TM}}$ Cell Analyzer.

Multi-caspase analysis. MSTO-211H and $\mathrm{H} 28$ were seeded on a $10-\mathrm{cm}$ dish and treated with various concentrations of LCA $(10,20$ and $40 \mu \mathrm{M})$ for $48 \mathrm{~h}$. Caspase activity was measured using the Multi-caspase cell kit (Merck Millipore) and analyzed by Muse ${ }^{\mathrm{TM}}$ Cell Analyzer. 
A<smiles>C=CC(C)(C)c1cc(/C=C/C(=O)c2ccc(O)cc2)c(O)cc1O</smiles>

Licochalcone A
B

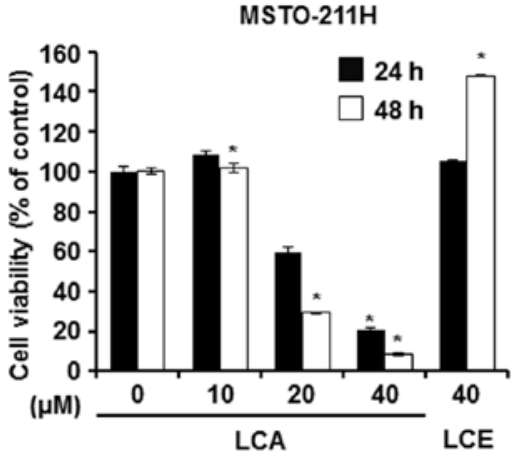

H28

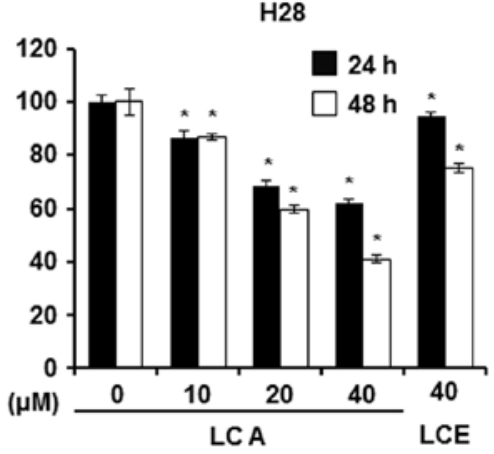

C
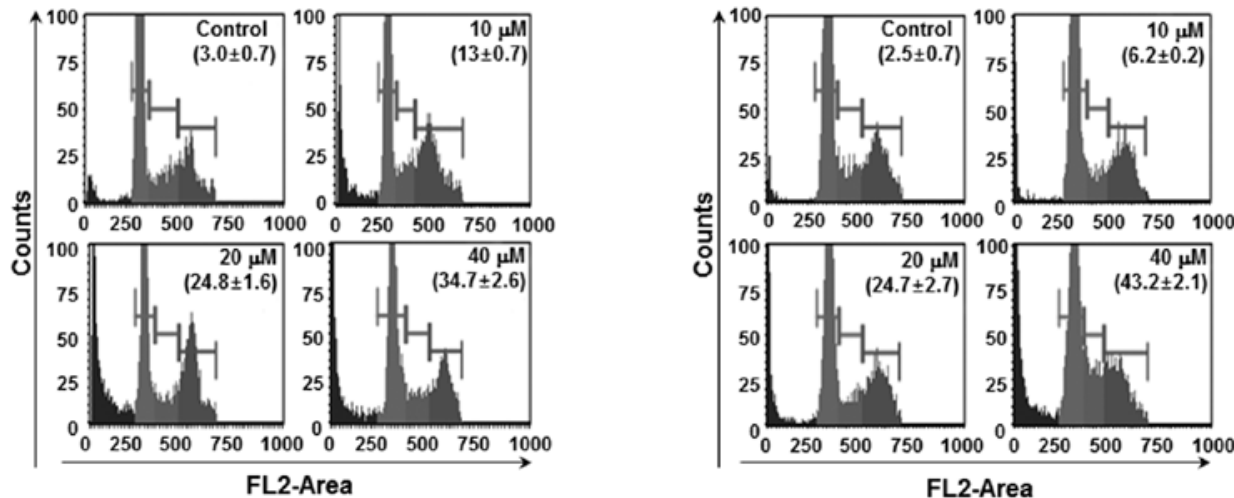

D
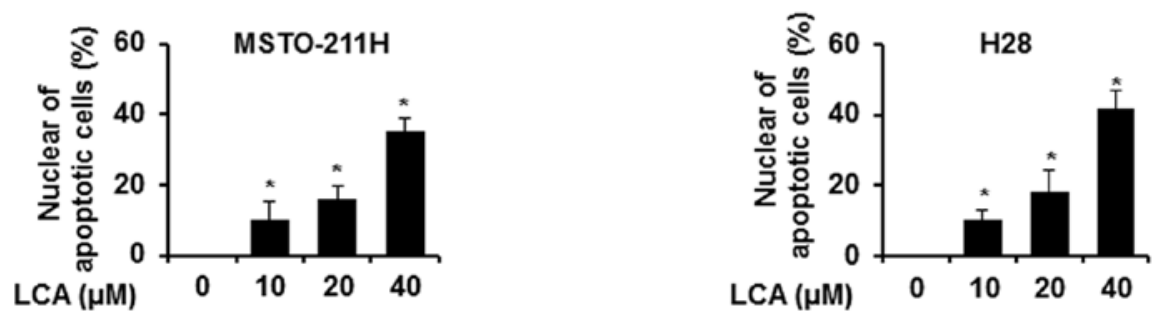

Figure 1. Effects of LCA on MPM cells viability. (A) Chemical structure of LCA. (B) Cell viability of MSTO-211H and H28 cells treated with LCA (10, 20 and $40 \mu \mathrm{M})$ and LCE $(40 \mu \mathrm{M})$, which was measured using MTS assay kits. (C) MSTO-211H and $\mathrm{H} 28$ cells were treated with 10, 20 and $40 \mu \mathrm{M} \mathrm{LCA}$ and the representative of sub- $\mathrm{G}_{1}$ population was measured by Muse analysis after cell cycle staining. (D) DNA fragmentation and nuclear condensation were quantified using DAPI staining. The data represent the mean percentage levels $\pm \operatorname{SD}(n=3) ;{ }^{*} p<0.05$, significant difference compared with DMSO-treated control cells by paired t-test.

Annexin V staining. The cells (MSTO-211H and H28) were seeded on a $10-\mathrm{cm}$ dish and treated with various concentrations of LCA $(10,20$ and $40 \mu \mathrm{M})$ for $48 \mathrm{~h}$. Both adherent and floating cells were harvested, washed once with PBS. For detection of apoptosis, cells were incubated with $\mathrm{Muse}^{\mathrm{TM}}$ Annexin V \& Dead Cell reagent (Merck Millipore) for 20 min at RT in the dark. Apoptotic and necrotic cells were analyzed by Muse ${ }^{\mathrm{TM}}$ Cell Analyzer.

Western blot analysis. The MSTO-211H and $\mathrm{H} 28$ cells were treated with LCA for $48 \mathrm{~h}$ and lysed in RIPA buffer (Intron Biotechnology, Seoul, Korea) containing a protease inhibitor. Protein concentrations were determined by BCA assay (Pierce Chemical, Rockford, IL, USA). Proteins were separated by SDS-PAGE and transferred to polyvinylidene fluoride (PVDF) membrane (GE Healthcare, Little Chalfont, UK). The membrane was blocked by 3 or $5 \%$ fat-free skim milk, then probed with primary and secondary antibodies. Bands of interest were visualized using ECL Western blotting detection regents (GE Healthcare).
Statistical analysis. The experimental data are expressed as the mean \pm standard deviation and statistical significance between different groups was determined using Student's t-test. Triplicate experiments were performed on the data obtained for the experimental groups. P-value of $<0.05$ was considered statistically significant.

\section{Results}

LCA inhibits cell growth of MPM. In order to investigate the anti-proliferative activity of licochalcone, MPM cells (MSTO$211 \mathrm{H}$ and $\mathrm{H} 28$ ) were treated with LCA or LCE. The structure of LCA is depicted in Fig. 1A. LCA and LCE were isolated from the roots of Glycyrrhiza inflata (20). Structure of LCA is similar to that of LCE, but the positions of the methyl groups of allyl group in LCA were different from those of LCE $(20,21)$. To determine inhibitory effects of LCA and LCE on cell viability, MTS assay was done after 24 and $48 \mathrm{~h}$. As a results, it was found that the $\mathrm{IC}_{50}$ value of LCA was 26 and $30.4 \mu \mathrm{M}$ in MSTO-211H and H28, respectively. LCA inhibited 

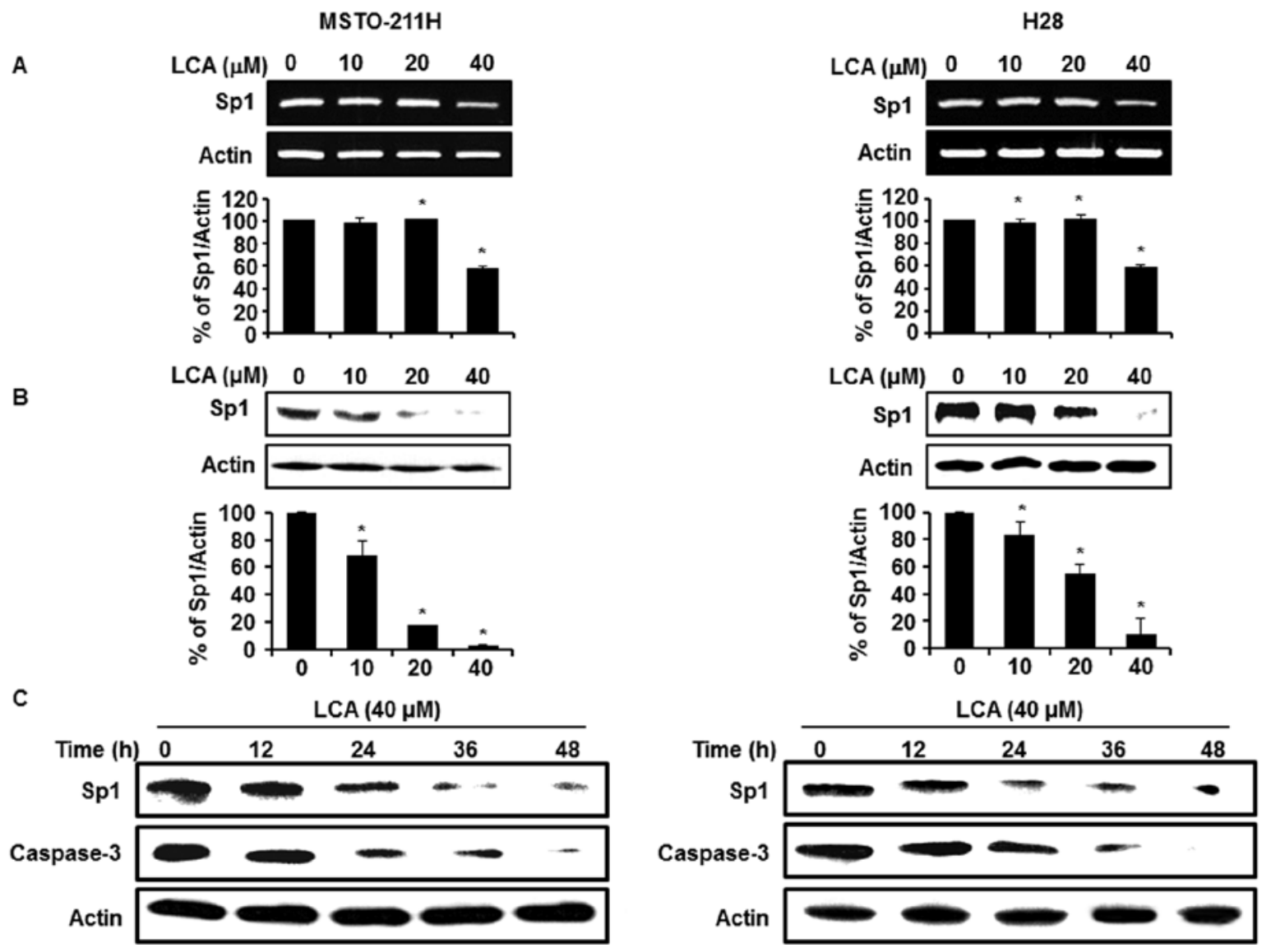

Figure 2. LCA suppresses Spl proteins in MPM cells undergoing apoptosis. MSTO-211H (left panel) and H28 (right panel) cells were treated with 10, 20 and $40 \mu \mathrm{M} \mathrm{LCA}$ for $48 \mathrm{~h}$. RT-PCR and western blot analysis were employed. The graphs indicate the mRNA (A) and protein (B) expression levels of Sp1 normalized to actin. (C) To examine the time-dependent effect of LCA on Sp1 and caspase-3, MSTO-211H and H28 cells were treated with $40 \mu \mathrm{M} \mathrm{LCA}$ for 12, 24, 36 and $48 \mathrm{~h}$. Actin was included as the loading control.

A

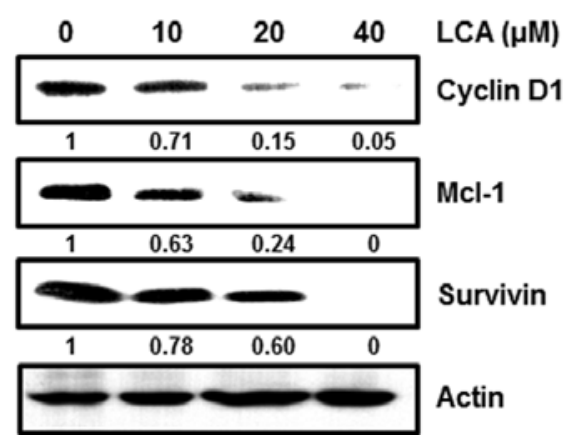

B

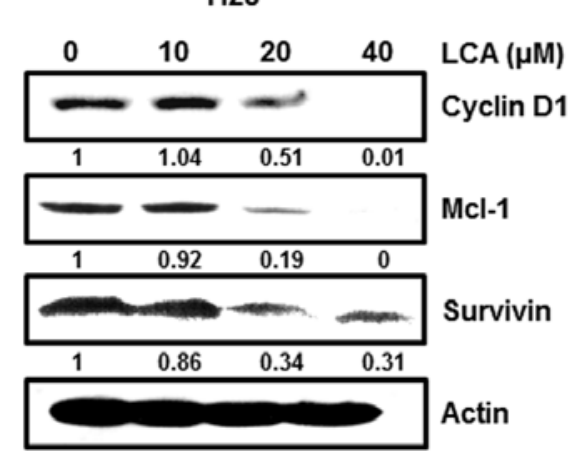

Figure 3. The effect of LCA on Sp1 downstream target proteins in: (A) MSTO-211H and (B) H28 cells treated with 10, 20 and $40 \mu$ M LCA. The effect of LCA on Cyclin D1, Mcl-1 and Survivin protein expression levels were determined by western blot analysis. The blots were re-probed with actin as a loading control.

cell survival of MSTO-211H cell in a dose-dependent manner, 101.6, 29.0 and 8.4\% over the concentrations used (10, 20 and $40 \mu \mathrm{M})$. Similarly, $\mathrm{H} 28$ cells represented cell viability of 86.7 , 59.7 and $41.0 \%$ at the concentrations used $(10,20$ and $40 \mu \mathrm{M})$, respectively. However, LCE had a weak cytotoxic activity in H28 but not MSTO-211H for 48 h (Fig. 1B).

LCA treatment regulates the expression of cell cycle arrest and induces apoptosis in MPM cells. In order to examine correlation of altered cell cycle to growth inhibitory effects of
LCA, we carried out PI staining of LCA-treated cells and then flow cytometric analyses. We analyzed cell cycle profiles of cells treated with LCA $(10,20$ and $40 \mu \mathrm{M})$ for $48 \mathrm{~h}$ by Muse ${ }^{\mathrm{TM}}$ Cell Analyzer. As a result, we observed that sub- $\mathrm{G}_{1}$ phase was increased in a dose-dependent manner by LCA, indicating that sub- $G_{1}$ phase was increased from $3.0 \pm 0.7$ to $34.7 \pm 2.6 \%$ for MSTO-211H (Fig. 1C, left) and was accumulated from 2.5 \pm 0.7 to $43.2 \pm 2.1 \%$ for $\mathrm{H} 28$ (Fig. 1C, right). LCA induced considerably fragmentation and condensation of DNA of MPM cells in a dose-dependent manner (Fig. 1D). Annexin V staining 
MSTO-211H

A

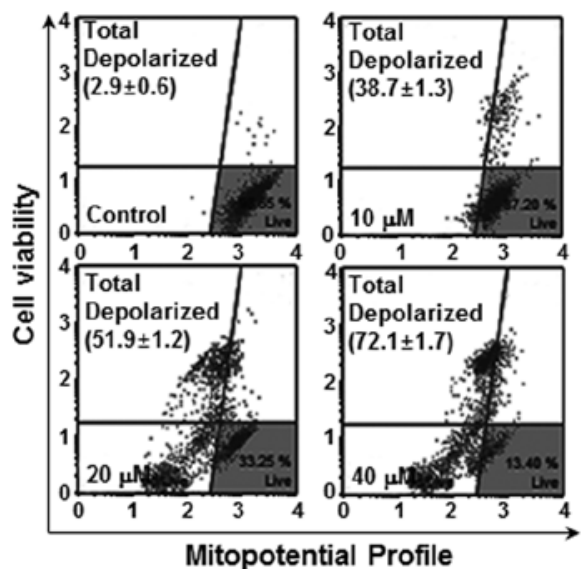

C

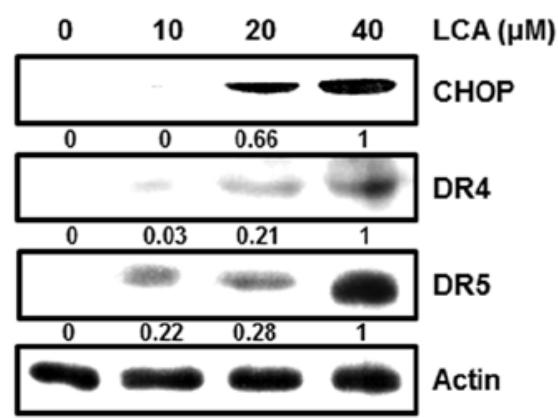

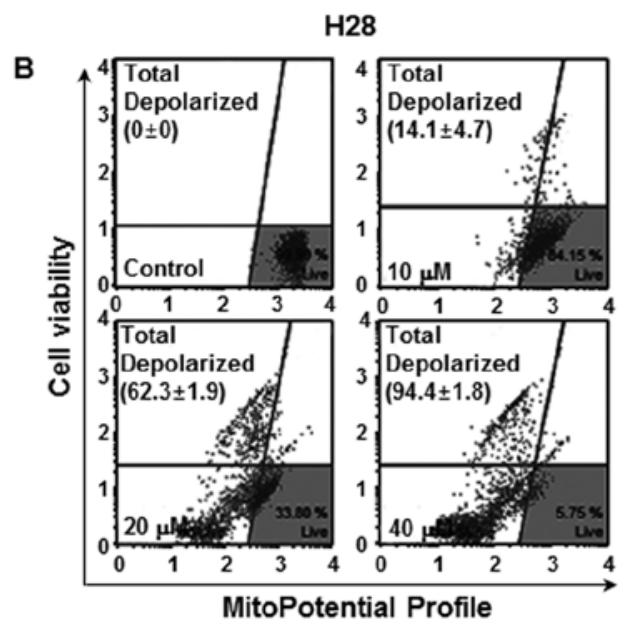

D

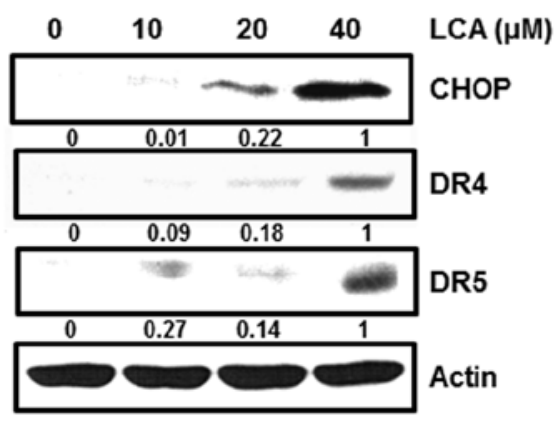

Figure 4. Induction of mitochondrial dysfunction and increased expression level of the death receptor protein in LCA induced apoptosis. The graph shows the process of depolarization of the mitochondrial membrane (A and B). The data represent the mean percentage levels $\pm \mathrm{SD}(\mathrm{n}=3)$. The expression levels of $\mathrm{CHOP}$ and death receptor proteins (DR4 and DR5) were analyzed by western blotting in MSTO-211H (C) and H28 (D) cells. Actin was used in the loading control.

indicated that phosphatidylserine was exposed on the outer leaflet of the membrane during cell death. Early apoptosis of cells was progressed to late apoptosis by LCA treatment. In MSTO-211H and $\mathrm{H} 28$, late apoptotic cell populations were increased from $2.8 \pm 1.5$ to $47.1 \pm 0.9 \%$ (Fig. $5 \mathrm{C}$ ) and from $2.7 \pm 0.1$ to $17.6 \pm 4.9 \%$, respectively (Fig. $5 \mathrm{D}$ ).

LCA modulates Sp1 protein level in MPM cells. To demonstrate the link between $\mathrm{Sp1}$ and cell death, expression levels of Sp1 were primarily examined by western blotting after treatment of MPM cells with LCA $(10,20$ and $40 \mu \mathrm{M})$. Fig. 2A and $\mathrm{B}$ show that Sp1 mRNA and protein expression levels were reduced by LCA in a dose-dependent manner. Also, we observed that the caspase- 3 associated with cell death was cleaved in a time-dependent manner (12, 24, 36 and $48 \mathrm{~h}$; Fig. 2C). Sp1 is a transcription factor that modulates cell cycle regulation, anti-proliferative and apoptotic cell death by regulating the promoter of the target gene. Sp1 regulates the expression of Cyclin D1, Mcl-1 and Survivin contributing to cell proliferation (23). In line with previous results, Sp1 target proteins are significantly reduced by LCA treatment (Fig. 3).

LCA affects the permeability of the mitochondrial membrane. Mitochondrial membrane potential is closely associated with Bcl-2 family-mediated apoptosis. To investigate whether LCA could affect the mitochondrial membrane potential, the degree of depolarization was measured by staining of MPM cells with 5,5',6,6'-tetrachloro-1,1',3,3'-tetraethylbenzimidazolylcarbocyanine iodide reagent. In MSTO-211H (Fig. 4A), total depolarized cell population was $38.7 \pm 1.3,51.9 \pm 1.2$ and $72.1 \pm 1.7 \%$ and in $\mathrm{H} 28$ it was $14.1 \pm 4.7,62.3 \pm 1.9$ and $94.4 \pm 1.8 \%$ in a dosedependent manner, respectively. We measured the levels of proteins associated with extrinsic apoptosis pathway (24). CHOP, which is increased by ER stress, activates TNF-related apoptosis-inducing ligand (TRAIL) death receptors DR4 and DR5 (25). The expression levels of CHOP, DR4 and DR5 were substantially increased in MSTO-211H (Fig. 4C) and H28 cells (Fig. 4D).

$L C A$ regulates apoptotic and anti-apoptotic molecules in MPM cells. To observe the molecular mechanisms of cell apoptosis in MSTO-211H (Fig. 5A) and H28 (Fig. 5B) cells, we examined the expression of target proteins. First, after LCA treatment $(10,20$ and $40 \mu \mathrm{M})$ the expression of pro- and anti-apoptotic proteins was investigated. As a result, BID, $\mathrm{Bcl}_{\mathrm{xL}}$ Caspase-3 and C-PARP expression were specifically decreased and cleaved in MPM cell lines. On the other hand, Bax expression of pro-apoptotic protein was increased. Multicaspase activity was increased in MPM cells treated with various concentrations of LCA $(10,20$ and $40 \mu \mathrm{M})$ for $48 \mathrm{~h}$ (Fig. 5E and F). Total multi-caspase activity in MSTO-211H (Fig. 5E) was $14.2 \pm 1.6,28.1 \pm 3.4$ and $81.3 \pm 0.7 \%$ that in $\mathrm{H} 28$ (Fig. $5 \mathrm{~F}$ ) was $16.7 \pm 0.5,32.1 \pm 2.9$ and $37.9 \pm 3.0 \%$ in a dosedependent manner, respectively. 
A

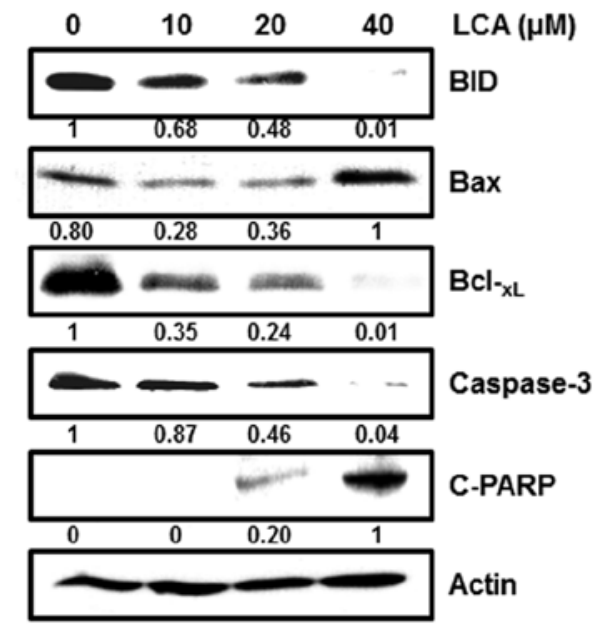

C

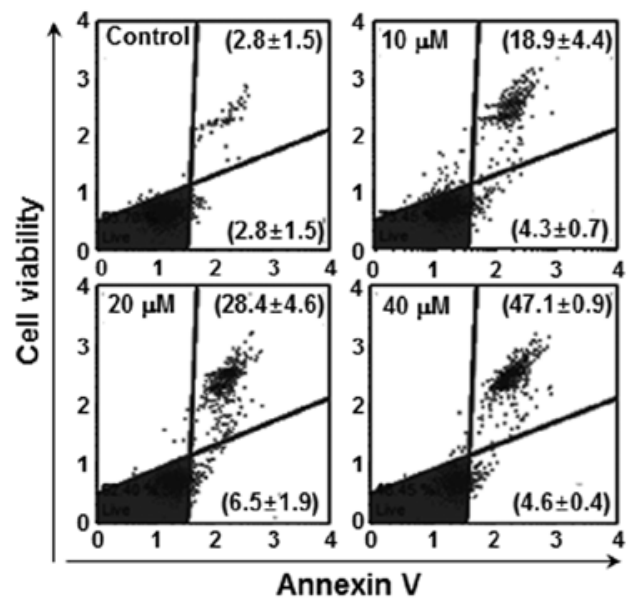

E

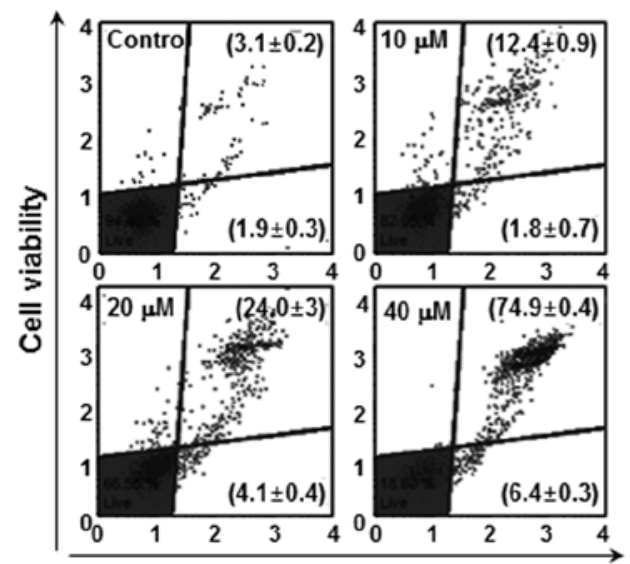

FL2-Area
B

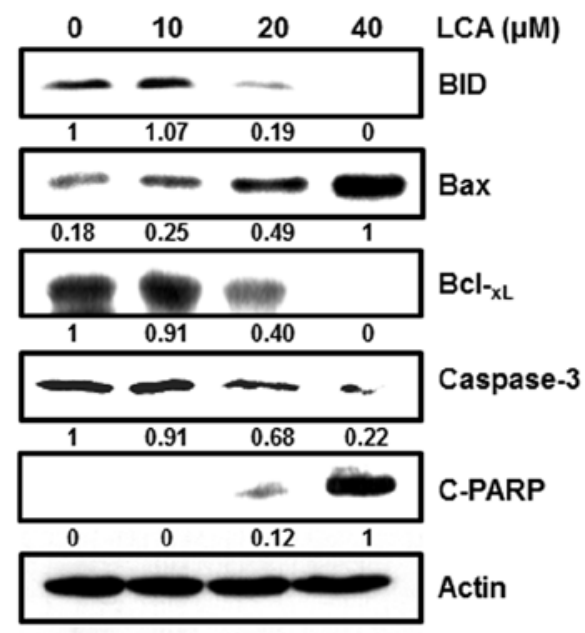

D

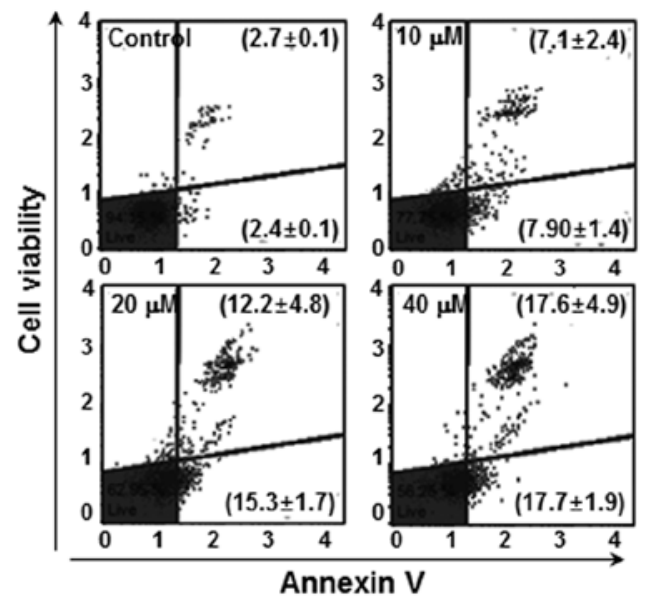

$\mathbf{F}$

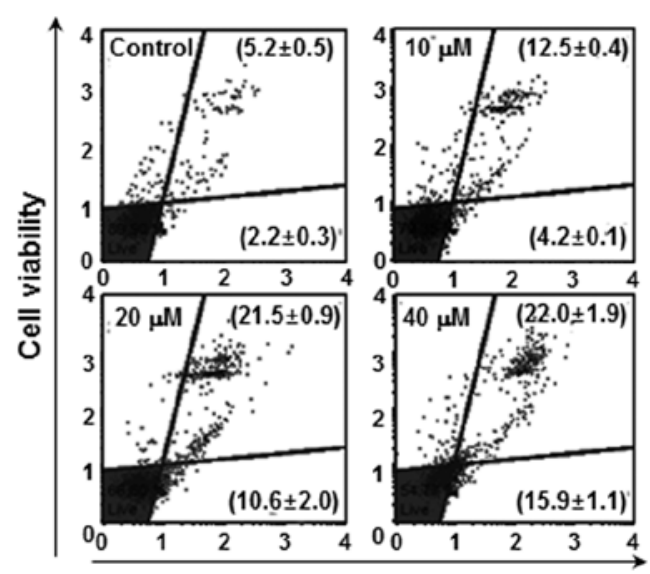

FL2-Area

Figure 5. The effect of LCA on expression of proteins associated with apoptosis in MPM cells. MSTO-211H (A) and H28 (B) cells were treated with 10, 20 and $40 \mu \mathrm{M} \mathrm{LCA}$ for $48 \mathrm{~h}$ and analyzed by western blot analysis using antibodies against $\mathrm{BID}, \mathrm{Bax}, \mathrm{Bcl}_{\mathrm{xL}}$, Caspase-3 and C-PARP. Actin was used to normalize the protein bands. Induction of apoptosis in MPM cells by LCA was quantitatively analyzed using flow cytometry. The plots were drawn by analyzing MSTO-211H (C and E) and H28 (D and F) cells after exposure to LCA 10, 20 and $40 \mu \mathrm{M}$ for $48 \mathrm{~h}$ with the Muse ${ }^{\mathrm{TM}}$ Cell Analyzer. The analyzer profiles were made after Annexin V (C and D) and multi-caspase staining (E and F). (C and D) Relative to the lower left, plots represent early apoptosis, late apoptosis/Dead and Dead in the counter-clockwise direction. (E and F) Lower left is a control group, each quadrant indicate caspase positive, caspase-positive/Dead and Dead in the counter-clockwise direction. The data represent the mean percentage levels \pm SD $(n=3)$.

\section{Discussion}

MPM is a cancer that develops in the mesothelium that covers many of the internal organs of the body (4). The most common anatomical location of mesothelioma is the pleura and it is most commonly caused by exposure to asbestos (26). Currently, surgery and radiotherapy have been recommended, but the prognosis is poor. Therefore, chemotherapy is gaining attention (27). 
LCA is a novel estrogenic flavonoid and a natural phenol product. It can be derived from roots of Glycyrrhiza inflata and shows beneficial activities such as anti-malarial, anticancer, anti-bacterial and anti-viral effects $(12,28)$. There is a growing body of evidence that it exhibits an anticancer effect, but it is not clear whether LCA would be effective against MPM. In this study, we investigated whether LCA could induce apoptosis via Sp1 in MPM cells (MSTO-211H and H28). First, MTS assay was performed to assess how the LCA could affect cell proliferation. Cell growth was inhibited in a concentration-dependent manner by LCA. In addition, we treated LCE $(40 \mu \mathrm{M})$ bearing the similar allyl group on different positions of methyl groups compared with LCA. In contrast to LCA, LCE was not effective in MPM cells $(21,29)$. The different allyl group of LCA might be critical for its anticancer activity against MPM. PI staining was performed to demonstrate that LCA had an anti-proliferative effect and induced apoptotic cell death. We checked the changes in percentage of sub- $\mathrm{G}_{1}$ fraction. Percentage of cells present in sub- $\mathrm{G}_{1}$ peak was increased. Annexin $\mathrm{V}$ staining and caspase activity were measured to further verify apoptotic cell death (30). Caspase activity as well as the numbers of early and late apoptotic cells was increased. Accordingly, it is evident that the cytostatic effect of LCA in MPM cells is due to induction of cell death.

To prove that apoptosis is mediated by regulation of $\mathrm{Sp} 1$ transcription factor, we examined the apoptosis-related factors using western blotting. Sp1 is a zinc finger transcription factor that binds to GC-rich motifs of many promoters (23) and it is involved in many cellular processes, including cell differentiation, cell growth, apoptosis, immune responses, response to DNA damage and chromatin remodeling (18). LCA downregulated expression levels of mRNA and protein level of the Sp1 in a concentration and time-dependent manner. Consistent with Sp1 levels, Sp1 regulatory proteins (Cyclin D1, Mcl-1 and Survivin) were also diminished, hypothesizing that the LCA induced cell death via downregulation of Sp1.

Pathologically, apoptosis is referred to as the normal process of active cell death of organisms to remove damaged or unnecessary cells. Mitochondria not only produced energy in cells but also regulates cell fate factors $(31,32)$. In this study, we revealed how LCA could be involved in the morphological control of mitochondria in MPM cells and further delineated the mechanisms associated with the induction of cell death.

Apoptosis involves two different pathways. One is the extrinsic pathway of caspase activation that is mediated by the cell surface death receptor and the other is the mitochondria initiated pathway $(33,34)$. Extrinsic pathway begins with the binding of ligands to a subset of the members of the TNF receptor superfamily (35-37). TRAIL is able to interact with the membrane receptor DR4/TRAIL-R1 and DR5/TRAIL-R2 bearing a different functional death domain $(38,39)$. An increase in expression of CHOP elevates the levels of membrane receptors DR4 and DR5, which operate cleavage of Bid into tBid. Cytochrome $C$ induces the activation of caspase- 8 and -9 , leading to downstream activation of caspase- 3 to promote the final cell death mechanism (35). We found that the expression of CHOP, DR4 and DR5 was upregulated while the levels of both caspase- 3 and Bid decreased in the LCA-treated MPM cells. These results suggest that LCA may be able to induce apoptosis in MPM cells via the receptor-mediated pathway. The second pathway of apoptosis involves the participation of mitochondria, regulated by the anti-apoptotic and pro-apoptotic members of the Bcl-2 family (40). Bcl-2 family is composed of pro-apoptotic proteins such as Bax, Bak and Bid and anti-apoptotic proteins such as Bcl-2, $\mathrm{Bcl}_{\mathrm{xL}}$ and $\mathrm{Mcl}-1$. Bax or Bak are bound to the mitochondria membrane, releasing cytochrome $\mathrm{C}$ into cytosol $(41,42)$. The released cytochrome $C$ in turn activates caspases, which transmit cell death signals by dividing PARP (43-46). Decreased expression of the cytosolic Bax and concomitant increase of the mitochondrial Bax indicated that LCA induced the translocation of Bax from cytosol to the mitochondrial membrane. Western blot results demonstrated that LCA upregulated the expression level of Bax and C-PARP and downregulated the level of Bcl-2 in MPM cells. In combination with the effect of LCA on mitochondrial membrane potential in MPM cells, these results indicate that LCA induces apoptosis in MPM cells via the mitochondria-mediated intrinsic pathway.

Taken together, this study reveals the relevance of Sp1, MPM, LCA and mitochondria to apoptosis. We demonstrated that LCA induced apoptosis by suppressing the expression of Sp1 in MPM cells. LCA-mediated Sp1 down-regulation induced morphological changes of mitochondria, leading to apoptotic cell death. Therefore, our studies strongly suggest a potential novel strategy to use LCA as an effective anticancer therapeutic agent for MPM patients with Sp1 overexpression.

\section{Acknowledgements}

This study was supported by Gloden Seed Project (2130051), Ministry of Agriculture, Food and Rural Affairs.

\section{References}

1. Siegel R, Ma J, Zou Z, et al: Cancer statistics, 2014. CA Cancer J Clin 64: 9-29, 2014.

2. Tiwari RC, Ghosh K, Jemal A, et al: A new method of predicting US and state-level cancer mortality counts for the current calendar year. CA Cancer J Clin 54: 30-40, 2004.

3. Ahmed I, Ahmed Tipu S and Ishtiaq S: Malignant mesothelioma. Pak J Med Sci 29: 1433-1438, 2013.

4. Linton A, Cheng YY, Griggs K, et al: An RNAi-based screen reveals PLK1, CDK1 and NDC80 as potential therapeutic targets in malignant pleural mesothelioma. Br J Cancer 110: 510-519, 2013.

5. Cunha P, Luz Z, Seves I, et al: Malignant peritoneal mesothelioma - diagnostic and therapeutic difficulties. Acta Med Port 15: 383-386, 2002 (In Portuguese).

6. de Pangher Manzini V: Malignant peritoneal mesothelioma. Tumori 91: 1-5, 2005.

7. Kusamura S, Deraco M, Baratti D, et al: Cytoreductive surgery followed by intra peritoneal hyperthermic perfusion in the treatment of peritoneal surface malignancies: morbidity and mortality with closed abdomen technique. J Exp Clin Cancer Res 22: 207-212, 2003

8. Rodriguez D, Cheung MC, Housri N, et al: Malignant abdominal mesothelioma: defining the role of surgery. J Surg Oncol 99: 51-57, 2009.

9. Yuan X, Li D, Zhao H, et al: Licochalcone A-induced human bladder cancer T24 cells apoptosis triggered by mitochondria dysfunction and endoplasmic reticulum stress. Biomed Res Int 2013: 474272, 2013.

10. Yao K, Chen $\mathrm{H}$, Lee $\mathrm{MH}$, et al: Licochalcone A, a natural inhibitor of c-Jun N-terminal kinase 1. Cancer Prev Res 7: 139-149, 2014.

11. Kim JK, Shin EK, Park JH, et al: Antitumor and antimetastatic effects of licochalcone A in mouse models. J Mol Med 88: 829-838, 2010.

12. Xiao XY, Hao M, Yang XY, et al: Licochalcone A inhibits growth of gastric cancer cells by arresting cell cycle progression and inducing apoptosis. Cancer Lett 302: 69-75, 2011. 
13. Yo YT, Shieh GS, Hsu KF, et al: Licorice and licochalcone-A induce autophagy in LNCaP prostate cancer cells by suppression of Bcl-2 expression and the mTOR pathway. J Agric Food Chem 57: 8266-8273, 2009.

14. Cui Y, Ao M, Li W, et al: Anti-inflammatory activity of licochalcone A isolated from Glycyrrhiza inflata. Z Naturforsch C 63 : 361-365, 2008 .

15. Funakoshi-Tago M, Tanabe S, Tago K, et al: Licochalcone A potently inhibits tumor necrosis factor alpha-induced nuclear factor-kappaB activation through the direct inhibition of IkappaB kinase complex activation. Mol Pharmacol 76: 745-753, 2009.

16. Li L and Davie JR: The role of Sp1 and Sp3 in normal and cancer cell biology. Ann Anat 192: 275-283, 2010.

17. Safe $\mathrm{S}$ and Abdelrahim M: Sp transcription factor family and its role in cancer. Eur J Cancer 41: 2438-2448, 2005.

18. Courey AJ and Tjian R: Analysis of Sp1 in vivo reveals multiple transcriptional domains, including a novel glutamine-rich activation motif. Cell 55: 887-898, 1988

19. Chuang JY, Wu CH, Lai MD, et al: Overexpression of Sp1 leads to p53-dependent apoptosis in cancer cells. Int J Cancer 125 : 2066-2076, 2009.

20. Yoon G, Jung YD and Cheon SH: Cytotoxic allyl retrochalcone from the roots of Glycyrrhiza inflata. Chem Pharm Bull 53 : 694-695, 2005

21. Fu Y, Chen J, Li YJ, et al: Antioxidant and anti-inflammatory activities of six flavonoids separated from licorice. Food Chem 141: 1063-1071, 2013.

22. Goldberg JL, Zanella CL, Janssen YM, et al: Novel cell imaging techniques show induction of apoptosis and proliferation in mesothelial cells by asbestos. Am J Respir Cell Mol Biol 17: 265-271, 1997.

23. Bouwman P and Philipsen S: Regulation of the activity of Sp1-related transcription factors. Mol Cell Endocrinol 195: 27-38, 2002.

24. Chen Q, Liu XF and Zheng PS: Grape seed proanthocyanidins (GSPs) inhibit the growth of cervical cancer by inducing apoptosis mediated by the mitochondrial pathway. PLoS One 9: e107045, 2014

25. Condamine T, Kumar V, Ramachandran IR, et al: ER stress regulates myeloid-derived suppressor cell fate through TRAILR-mediated apoptosis. J Clin Invest 124: 2626-2639, 2014.

26. Gultekin KE, Yurdakonar MK, Yaman E, et al: Effects of cisplatin and panobinostat on human mesothelial (Met-5A) and malignant pleural mesothelioma (MSTO-211H) cells. Genet Mol Res 12: 5405-5413, 2013

27. Lee YJ, Park IS, Lee YJ, et al: Resveratrol contributes to chemosensitivity of malignant mesothelioma cells with activation of p53. Food Chem Toxicol 63: 153-160, 2013.

28. Tsai JP, Hsiao PC, Yang SF, et al: Licochalcone A suppresses migration and invasion of human hepatocellular carcinoma cells through downregulation of MKK4/JNK via NF-kappaB mediated urokinase plasminogen activator expression. PLoS One 9: e86537, 2014.

29. Park HG, Bak EJ, Woo GH, et al: Licochalcone E has an antidiabetic effect. J Nutr Biochem 23: 759-767, 2012
30. Chang SF, Chang CA, Lee DY, et al: Tumor cell cycle arrest induced by shear stress: Roles of integrins and Smad. Proc Natl Acad Sci USA 105: 3927-3932, 2008.

31. Li H, Zhu H, Xu CJ, et al: Cleavage of BID by caspase 8 mediates the mitochondrial damage in the Fas pathway of apoptosis. Cell 94: 491-501, 1998

32. Kaufmann T, Strasser A and Jost PJ: Fas death receptor signalling: roles of Bid and XIAP. Cell Death Differ 19: 42-50, 2012

33. Teng CL, Han SM, Wu WC, et al: Mechanistic aspects of lauryl gallate-induced differentiation and apoptosis in human acute myeloid leukemia cells. Food Chem Toxicol 71: 197-206, 2014

34. Law CK, Kwok HH, Poon PY, et al: Ginsenoside compound K induces apoptosis in nasopharyngeal carcinoma cells via activation of apoptosis-inducing factor. Chin Med 9: 11, 2014.

35. Fossati S, Ghiso J and Rostagno A: TRAIL death receptors DR4 and DR5 mediate cerebral microvascular endothelial cell apoptosis induced by oligomeric Alzheimer's Abeta. Cell Death Dis 3: e321, 2012.

36. Haase G, Pettmann B, Raoul C, et al: Signaling by death receptors in the nervous system. Curr Opin Neurobiol 18: 284-291, 2008.

37. Wilson NS, Dixit V and Ashkenazi A: Death receptor signal transducers: nodes of coordination in immune signaling networks. Nat Immunol 10: 348-355, 2009.

38. Rizzardi C, Leocata P, Ventura L, et al: Apoptosis-related factors (TRAIL, DR4, DR5, DcR1, DcR2, apoptotic cells) and proliferative activity in ameloblastomas. Anticancer Res 29: 1137-1142, 2009.

39. Almasan A and Ashkenazi A: Apo2L/TRAIL: apoptosis signaling, biology and potential for cancer therapy. Cytokine Growth Factor Rev 14: 337-348, 2003.

40. Martin SJ and Green DR: Protease activation during apoptosis: death by a thousand cuts? Cell 82: 349-352, 1995.

41. Jin Z and El-Deiry WS: Overview of cell death signaling pathways. Cancer Biol Ther 4: 139-163, 2005.

42. Kim EJ, Lee YJ, Shin HK, et al: Induction of apoptosis by the aqueous extract of Rubus coreanum in HT-29 human colon cancer cells. Nutrition 21: 1141-1148, 2005.

43. Oliver FJ, de la Rubia G, Rolli V, et al: Importance of poly(ADPribose) polymerase and its cleavage in apoptosis. Lesson from an uncleavable mutant. J Biol Chem 273: 33533-33539, 1998.

44. Wong WW and Puthalakath $\mathrm{H}$ : Bcl-2 family proteins: the sentinels of the mitochondrial apoptosis pathway. IUBMB Life 60: 390-397, 2008

45. El-Hamoly T, Hegedus C, Lakatos P, et al: Activation of poly(ADP-ribose) polymerase-1 delays wound healing by regulating keratinocyte migration and production of inflammatory mediators. Mol Med 20: 363-371, 2014.

46. Toth-Zsamboki E, Horvath E, Vargova K, et al: Activation of poly(ADP-ribose) polymerase by myocardial ischemia and coronary reperfusion in human circulating leukocytes. Mol Med 12: $221-228,2006$ 